\title{
Comments on the generation mechanism of Seismic Electric Signals
}

\author{
E. Dologlou \\ Solid State Section, Department of Physics, University of Athens, Panepistimiopolis, Zografos 15784 Athens, Greece
}

Received: 27 June 2011 - Accepted: 12 September 2011 - Published: 2 December 2011

\begin{abstract}
Recent laboratory measurements on rocks under varying pressure lead to results which strengthen a model suggested by the author for the explanation of the power law relation that interconnects the lead time of Seismic Electric Signals and earthquake stress drop. In addition, recent applications of a thermodynamic model that interrelates the defect parameters in materials of geophysical interest and their bulk properties open a new window to further advance the aforementioned explanation.
\end{abstract}

\section{Introduction}

During the last years, fracture in the Earth's heterogeneous crust and consequently preparation of earthquakes are predicted by the concept of criticality (Bak, 1996; Tucotte, 1997; Sornette, 2000; Rundle et al., 2000; Kossobokov et al., 2002). Nonlinear procedures that obey power laws and exhibit fractal geometry (Telesca et al., 2005; Teotia and $\mathrm{Ku}-$ mar, 2007, 2011) prevail in the pre-focal area, which seismicity upon increasing tectonic stress, evolves from microfracture to mega-fault (Telesca et al., 2003a, b). Since criticality governs the development of large earthquakes, one can expect that its associated precursors may signal the critical approach of the impending earthquake.

Three decades of experimental data showed that when the increasing tectonic stress in the pre-seismic region reaches a critical value $P_{\text {cr }}$, a seismic electric signal, known as SES, is emitted from the candidate area and is recognized as a low frequency $(1 \mathrm{~Hz})$ transient change of the Earth's telluric field which is monitored by a telemetric network over continental Greece (Varotsos and Alexopoulos, 1984a, b; Varotsos and Lazaridou, 1991; Varotsos et al., 1986, 1993a, b). Further

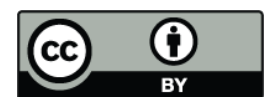

Correspondence to: E. Dologlou (edologl@phys.uoa.gr) increase of pressure finally leads to the rock failure and the occurrence of the earthquake. Thus, the emission of the SES preceding the rupture signals that the pre-seismic region enters the critical stage where dynamic nonlinear processes prevail.

Power law relations between earthquake parameters and associated precursory SES, with exponents falling in the range of critical values, have been found. These have been explained on the basis of a model proposed by Dologlou $(2008,2009,2010)$. It is the objective of this paper to draw attention to the point that new laboratory experimental data (Papathanasiou et al., 2010) considerably support and further strengthen this model.

\section{Compatibility of the proposed model with new experimental results}

Possible interconnection between SES lead time, which is the time difference $\Delta t$, between the SES detection and the earthquake occurrence (Varotsos and Alexopoulos, 1984a, b) and earthquake stress drop $\Delta \sigma$ (difference between two states of stress at a point on a fault before and after rupture) has been examined in various papers. Power law relations given by the formula

$\Delta \sigma_{\mathrm{B}}=k \Delta t^{-\alpha}$

with $k$ constant and exponent $\alpha=0.32$ lying in the range of critical exponents for fracture, (Sornette et al.,1989; Sornette and Sornette, 1990) has been obtained (Dologlou, 2008, 2009, 2010).

The values of lead time have been recently established in view of the possibility that when employing natural time (Varotsos et al., 2002, 2005), the occurrence time of an impending earthquake can be determined (Varotsos et al., 2006a, b). 
Although, a tentative model to explain the physical meaning of the observed correlations was suggested, two points which will be discussed below, remained open for further clarification. One is related to the basic assumption for the emission of a SES such as the existence of rocks in the earth's crust with negative migration volume and the other concerns the hypothesis that the difference, between the rock fracture stress required for the earthquake occurrence, $P_{\mathrm{fr}}$, and the critical stress for the SES emission $P_{\text {cr }}$ in the area of the impending earthquake can approximately express the stress drop.

Let us first present the mechanism for the SES emission. The predominant process of the SES generation is based on pressure stimulated currents (PSC) and is briefly described below:

Rocks in the Earth's crust contain various solids with intrinsic (Varotsos and Alexopoulos, 1978, 1979, 1984c) and extrinsic lattice defects. Particularly, in ionic solids doped with aliovalent impurities, vacancies appear for reasons of charge compensation (Kostopoulos et al., 1975; Varotsos et al., 1978) and electric dipoles are formed between these defects and nearby impurities. Upon increasing pressure the dipoles change orientation with a relaxation time $\tau$ according to the relation:

$\tau=(\lambda \nu)^{-1} \exp \left(g^{\text {act }} / k T\right)$

where $v$ is the attempt frequency for a jump to a number of $\lambda$ accessible paths in the vacancy vicinity, $T$ is the temperature and $g^{\text {act }}$ the Gibbs activation energy.

Before an earthquake, the increase of the tectonic stress in the future focal area affects not only some bulk properties of rocks, but also the thermodynamic parameters for activation of the defects in the included solids. Thus, if the activation volume $v^{\text {act }}$, expressed by the formula

$v^{\text {act }}=\left(d g^{\text {act }} / d P\right)_{T}$

is negative $v^{\text {act }}<0$ (Varotsos and Alexopoulos, 1980), an increase of pressure results in a decrease of the relaxation time $\tau$. When the stress in the candidate seismic area reaches a critical value $P_{\mathrm{cr}}$, a cooperative re-orientation of dipoles is achieved which causes the emission of a transient PSC current, known as SES signal (Varotsos and Alexopoulos, 1986) and then the following relation holds:

$\frac{b v^{\text {act }}}{k T}=-\frac{1}{\tau\left(P_{\mathrm{cr}}\right)}$

where $b(=\mathrm{dP} / \mathrm{dt})$ is the rate of the gradual pressure increase. A further increase of stress up to the fracture stress, $P_{\mathrm{fr}}$ will cause the failure of the rock and eventually the earthquake.

The basic condition of $v^{\text {act }}<0$ which is required for the emission of the SES was the crucial point of the suggested model since negative activation volumes were experimentally found in a few materials either by studying the pressure dependence of the electrical conductivity (in NAFION hydrogels (Fontanella et al., 1996) or the pressure dependence of the dielectric relaxation (in $\beta-\mathrm{PbF}_{2}$ doped with lanthanum (Fontanella et al., 1982), and semi-conducting polypyrrole (Papathanassiou et al., 2006, 2007). The assumption that probably such negative activation volumes also exist in rocks which could justify the SES generation was lacking experimental laboratory confirmation up to now. Very recently, laboratory measurements showed that negative $v^{\text {act }}$ values for "dipole" relaxation are obtained in hydrated alkaline earth carbonate rocks, such us high purity leukolite $\left(\mathrm{MgCO}_{3}\right)$ from Euboea, Greece and kataclastic limestone (polycrystalline $\mathrm{CaCO}_{3}$ ) from Greece that are commonly found in faults where earthquakes are most likely to occur. The leukolite and limestone samples were hydrated in the laboratory by sinking them in distilled water at $70^{\circ} \mathrm{C}$ for 4 days in order to achieve water saturation (Papathanassiou et al., 2010).

Hydration conditions of rock samples in the laboratory and rocks in situ, may be comparable according to the following argument.

When the heterogeneous rocks in the pre-seismic volume are subjected to increasing stress, cracking occurs locally and micro-fractures are progressively created inducing dilatancy. Differential stress exerts both static and dynamic effects on rock-mass permeability, modulating fluid flow in the region and water for the pressurized surrounding rock masses rushes to fill the fresh cracks that appear during the critical preearthquake stage (Surkov, 2002).

Thus, the existence of rocks with $v^{\text {act }}<0$ in the pre-focal area is realistic and, therefore, the basic condition for the SES emission seems to be satisfactorily fulfilled.

Concerning now the second topic which deals with the physics behind the interconnection of stress drop and lead time still remain unclear. In short, the lead time $\Delta t$ between the emission of the SES and the earthquake given by

$\Delta t=\left(P_{\mathrm{fr}}-P_{\mathrm{cr}}\right) / b$

(where $b$ is the stress accumulation rate) decreases for rapid stress increase (larger $b$ ) during the last preparatory earthquake stage (Varotsos et al., 1993a; Dologlou, 2010). A possible interrelation between the quantity $\left(P_{\mathrm{fr}}-P_{\mathrm{cr}}\right)$ and the stress drop of the earthquake is still difficult to prove although the obtained experimental data power law relation $\Delta \sigma_{\mathrm{B}} \propto \Delta \mathrm{t}{ }^{-\alpha}$, with critical exponent $\alpha=0.32$, (Dologlou, 2010) should be expected since it refers to dynamic critical processes. However, the following possibility seems to emerge in view of the recent advances: It is well known that point, as well as linear defects, govern the fracture of the materials and, thus, the value of $P_{\mathrm{fr}}$. (Recall that $P_{\mathrm{cr}}$ is interrelated with migration process only). The defect formation and migration energies are interconnected with bulk properties through a thermodynamical model termed $\mathrm{cB} \Omega$ model (Varotsos, 1976, 1977, 2007; Varotsos and Alexopoulos, 1984c). This states that the Gibbs formation $(f)$ energy $g^{f}$ and the Gibbs migration $(m)$ energy $g^{m}$ are proportional 
to the isothermal bulk modulus and to the mean volume per atom. In particular, $g^{f}=c^{f} B \Omega$ and $g^{m}=c^{m} B \Omega$ where $c^{f}$ and $c^{m}$ approximately constants. Furthermore, this model predicts that the ratio $v^{i} / g^{i}$, where $i$ refers to the formation $(i=f)$ and migration $(i=m)$ processes scales with $(d B / d P-1) / B$ where $d B / d P$ denotes the pressure derivative of the bulk modulus. The validity of this model has been already checked for the electric signals upon gradually increasing the stress in ionic crystals (Varotsos, 1977; Varotsos et al., 1999), but only very recently has it been applied with reasonable results in materials that govern geophysical processes (Zhang et al., 2011). In view of the latter result, future research should refer to electric signals emitted from materials of geophysical interest.

\section{Conclusions}

In this work, we attempt to enlighten two important points that remained unjustified in the proposed model by the author for the explanation of the underlying physics in power law relations found between the lead time of precursory Seismic Electric Signals and earthquake stress drop. The first point, is related to the crucial assumption for the emission of a SES, which requires the existence of rocks with negative activation volume in the pre-focal region. This topic was successfully clarified by recent laboratory results confirming that rock samples that are abundant in the earth's crust, under varying pressure and with hydration conditions comparable to those prevailing in the candidate focal area, do have negative activation volume.

The second point, which deals with the possibility that the difference between fracture and critical stress, $\left(P_{\mathrm{fr}}-P_{\mathrm{cr}}\right)$, may be related to the stress drop, still remains unclear. However, recent applications of a thermodynamic model that interconnects the defect parameters in materials of geophysical interest and their bulk properties open a new prospective for further research in this field.

Edited by: M. E. Contadakis

Reviewed by: two anonymous referees

\section{References}

Bak, P.: How Nature Works: The Science of Self-Organized Criticality, Springer-Verlag, New York, 212 pp., 1996.

Dologlou, E.: Possible relationship between Seismic Electric Signals (SES) lead time and earthquake stress drop, Proc. Jpn. Acad., Ser. B, 84, 117-122, 2008.

Dologlou, E.: Power law relationship between parameters of earthquakes and precursory electrical phenomena revisited, Nat. Hazards Earth Syst. Sci., 9, 17-23, doi:10.5194/nhess-9-17-2009, 2009.

Dologlou, E.: Power law relationship between parameters of earthquakes and precursory electrical phenomena revisited II, Nat.
Hazards Earth Syst. Sci., 10, 1403-1409, doi:10.5194/nhess-101403-2010, 2010.

Fontanella, J. J., Wintersgill, M. C., Figueroa, D. R., Chadwick, A. V., and Andeen, C. C.: Anomalous pressure dependence of dipolar relaxation in rare earth doped lead fluoride, Phys. Rev. Lett., 51, 1892-1895, 1982.

Fontanella, J. J., Edmondson, C. A., Wintersgill, M. C., Wu, Y., and Greenbaum, S. G.: High pressure electrical conductivity and NMR studies in variable equivalent weight NAFION membranes, Macromolecules, 29, 4944-4951, doi:10.1021/ma9600926, 1996.

Kossobokov, V. G., Keilis-Borok, V. I. Turcotte, D. L., and Malamud, B. D.: Implications of a statistical physics approach to earthquake hazard assessment and forecasting, Pure Appl. Geophys., 157, 2323-2349, 2002.

Kostopoulos, D., Varotsos, P., and Mourikis, S.: Conductivity of crystalline NaI, Can. J. Phys., 53, 1318-1320, 1975.

Papathanassiou, A. N., Sakellis, I., and Grammatikakis, J.: Separation of electric charge flow mechanisms in conducting polymer networks under hydrostatic pressure, Appl. Phys. Lett., 89, 222905, doi:10.1063/1.2768623, 2006.

Papathanassiou, A. N., Sakellis, I., and Grammatikakis, J.: Migration volume for polaron dielectric relaxation in disorderedmaterials, Appl. Phys. Lett., 91, 202103, doi:10.1063/1.2812538, 2007.

Papathanasiou, N. A., Sakellis, J., and Grammatikakis, C.: Negative activation volume for dielectric relaxation in hydrated rocks, Tectonophysics, 490, 307-309, 2010.

Rundle, B. J., Turcotte, D. L., and Klein, W.: Geocomplexity and the Physics of Earthquakes, Am. Geophys. Union, Washington, DC, 284 pp., 2000.

Sornette, D., Lagier, M., Roux, S., and Hansen, A.: Critical piezoelectricity in percolation, J. Physique, 50, 2201-2216, 1989.

Sornette, A. and Sornette, D.: Earthquake rupture as a critical point: consequences for telluric precursors, Tectonophysics, 179, 327334, 1990.

Sornette, D.: Critical Phenomena in Natural Sciences. Chaos,Fractals, Selforganization and Disorder: Concepts and Tools, Springer Ser. Synerg., Heidelberg, Springer-Verlag, New York, 423 pp., 2000.

Surkov, V., Uyeda, S., Tanaka, H., and Hayakawa, M.: Fractal Properties of medium and seismoelectric phenomena, J. Geodynamics, 33, 477-487, 2002.

Telesca, L., Lapenna, V., and Macchiato, M.: Spatial variability of the time correlated behaviour in Italian Seismicity, Earth Planet Sc. Lett., 213, 279-290, doi:10.1016/S0012-821X(03)00286-3, 2003.

Telesca, L., Lapenna, V., and Macchiato, M.: Multifractal fluctuations in seismic interspike series, Physica A, 354, 629-640, doi:10.1016/j.physa.2005.02.053, 2005.

Teotia, S. S. and Kumar, D.: The great Sumatra-Andaman earthquake of 26 December 2004 was predictable even from seismicity data of $\mathrm{mb}_{4.5}$ : A lesson to learn from nature, Indian J. Mar. Sci., 36, 122-127, 2007.

Teotia, S. S. and Kumar, D.: Role of multifractal analysis in understanding the preparation zone for large size earthquake in the North-Western Himalaya region, Nonlin. Processes Geophys., 18, 111-118, doi:10.5194/npg-18-111-2011, 2011.

Turcotte, D. L.: Fractals and Chaos in Geology and Geophysics, 2nd Edn., Cambridge University Press, 385 pp., 1997. 
Varotsos, P.: Comments on the formation entropy of a Frenkel defect in $\mathrm{BaF}_{2}$ and $\mathrm{CaF}_{2}$, Phys. Rev. B., 13, 938, 1976.

Varotsos, P. A.: On Temperature and pressure dependence of defect formation volume in ionic crystals, J. Phys. (France), 38, L455L458, 1977.

Varotsos, P.: Comparison of models that interconnect point defect parameters, J. Appl. Phys., 101, 123503, doi:10.1063/1.2745359, 2007.

Varotsos, P. and Alexopoulos, K.: The curvature in conductivity plots of silver halides as a consequence of anharmonicity, J. Phys. Chem. Solids, 39, 759-761, 1978.

Varotsos, P. and Alexopoulos, K.: On the possibility of the enthalpy of a Schottky defect decreasing with increasing temperature, J. Physics C, Solid State, 12, L761-L764, 1979.

Varotsos, P. and Alexopoulos, K.: Negative activation volumes of defects in solids, Phys. Rev. B, 21, 4898-4899, 1980.

Varotsos, P. and Alexopoulos, K. : Physical properties of the variations of the electric field of the earth preceding earthquakes, I, Tectonophysics, 110, 73-98, 1984a.

Varotsos, P. and Alexopoulos, K.: Physical properties of the variations of the electric field of the earth preceding earthquakes, II. Determination of epicentre and magnitude, Tectonophysics, 110, 99-125, 1984b.

Varotsos, P. and Alexopoulos, K.: Connection between the formation volume and formation Gibbs energy in noble gas solids, Phys. Rev. B, 30, 7305-7306, 1984c.

Varotsos, P. and Alexopoulos, K.: Thermodynamics of Point Defects and their Relation with the Bulk Properties, North Holland, Amsterdam 1986.

Varotsos, P. and Lazaridou, M.: Latest aspects of earthquake prediction in Greece based on seismic electric signals. I, Tectonophysics , 188, 321-347, 1991.
Varotsos, P., Ludwig W., and Alexopoulos, K.: Calculation of the formation volume of vacancies in solids, Phys. Rev. B, 18, 26832691, 1978.

Varotsos, P., Alexopoulos, K., Nomicos, K., and Lazaridou, M.: Earthquake prediction and electric signals, Nature, 322, 120, 1986.

Varotsos, P., Alexopoulos, K., and Lazaridou M.: Latest aspects of earthquake prediction in Greece based on Seismic Electric Signals II, Tectonophysics, 224, 1-37, 1993a.

Varotsos, P., Sarlis, N., and Lazaridou, M.: Interconnection of defect parameters and stress induced signals in ionic crystals, Phys. Rev. B., 59, 24-27, 1999.

Varotsos, P. A., Sarlis, N. V., and Skordas, E. S.: Long-range correlations in the electric signals that precede rupture, Phys. Rev. E, 66, 011902, 7 pp., 2002.

Varotsos, P., Alexopoulos, K., Lazaridou, M., and Nagao, T.: Earthquake predictions issued in Greece by seismic electric signals since February 6, 1990, Tectonophysics, 244, 269-288, 1993b.

Varotsos, P. A., Sarlis, N. V., Skordas, E. S., and Lazaridou, M.: Natural entropy fluctuations discriminate similar looking electric signals emitted from systems of different dynamics, Phys. Rev. E, 71, 011110, 11 pp., 2005.

Varotsos, P., Sarlis, N., Skordas, E., Tanaka, H., and Lazaridou, M.: Entropy of seismic electric signals: analysis in natural time under time reversal, Phys. Rev. E, 73, 031114, 8 pp., 2006 a.

Varotsos, P., Sarlis, N., Skordas, E., Tanaka, H., and Lazaridou, M.: Attempt to distinguish long-range temporal correlations from the statistics of the increments by natural time analysis, Phys. Rev. E, 74, 021123, 12 pp., 2006b.

Zhang, B., Wu, X., and Zhou, R.: Calculation of oxygen selfdiffusion coefficients in $\mathrm{Mg}_{2} \mathrm{SiO}_{4}$ polymorphs and $\mathrm{MgSiO}_{3}$ perovskite based on the compensation law, Solid State Ionics 186, 20-28, 2011. 